\title{
Tracer Assisted Passive Named Data Routing for Underwater Sensor Networks
}

\author{
Robert Martin, Jun-Hong Cui, Song Han \\ Department of Computer Science and Engineering, University of Connecticut, Storrs, CT, USA \\ \{robert.martin, jcui, song\}@engr.uconn.edu
}

\begin{abstract}
With the ever expanding area of Underwater Sensor Networks (UWSN), many networking challenges have yet to be fully analyzed and addressed. Some of the specific challenges include limited energy resources, node mobility, and broadcast limitations. More importantly, in bigger UWSN with large amounts of sensed data, bottlenecks form at relay nodes transferring packets to the surface. Additionally, this data in transition can be redundant and unimportant. Due to the harsh underwater environment, sensed data location and best routing path can constantly change. To help alleviate some of these challenges we propose Tracer assisted Passive Named Data Routing (TPNDR) protocol. This protocol uses tracers to form adaptive and robust forwarding tables based on data categorization. In this way, TPNDR is able to locate unknown data location and fetch requested data to the surface, without a flooding phase. From the use of timers we ensure that sensed data does not become stale over time. Through simulation of TPNDR, we are able to show improved energy efficiency and routing effectiveness.
\end{abstract}

Keywords—named data network; underwater sensor network; passive routing.

\section{INTRODUCTION}

Underwater Sensor Networks (UWSN) have received a great deal of attention from a broad range of areas in research over the past decade. This specific networking paradigm still suffers from the many challenges characterized by its structure, such as limited energy availability, large propagation delay, and node mobility [1, 2, 3]. Alongside these standard limitations is the ever-expanding notion of data becoming larger and more difficult to deal with given the complexity of better sensors. For UWSN, it is especially prevalent with large data being sensed that we see a bottleneck in our routing capabilities. Furthermore, we must fully address the key limitations to this bottleneck in order to properly propose a solution.

Current UWSN routing schemes rely on finding the shortest path, while trying to be energy conservative, to transmit data from the sensing point to the surface buoy or Autonomous Underwater Vehicle (AUV). In either case the network can transmit redundant data which can waste the already constrained resources. Therefore, in large networks with large amounts of sensed data (e.g. high resolution images) we must ensure that all packets being transmitted to the surface are relevant to the end user and arrive in a timely manner. UWSN are used in a broad range of applications, so we imply the idea

This work is supported in part by the US National Science Foundation, under Grant No.1018422, Grant No.1127084, Grant No.1128581, Grant No.1205665, Grant No.1208499, and Grant No.1331851, and the US Department of Education, under Grant No.P200A100141 and Grant No.P200A090342. of relevant data as anything that will assist the current research or job at hand. An example of this network would be a $\mathrm{CO}^{2}$ seafloor monitoring system which consists of densely spread sensors to cover a $5 \mathrm{~km}^{2}$ area. By assuming new data is sensed in frequent intervals of every few minutes, as well as some of the modem relay challenges given in [4], we see a relatively large bottleneck form in relay nodes transferring data packets to the surface. Based on acoustic propagation speeds (approximately $1500 \mathrm{~m} / \mathrm{s}$ ) among UWSN, we can assume that collected data will exceed the limitations of the network, thus requiring a new form of information storing and routing. A simple solution in this case would be inquiring a specific fetch and retrieval type of routing protocol. This, of course, is again limited due to the current IP-based protocols in place and the dynamic characteristics of UWSN. While certain nodes can be stationary, sensed data location and the best routing path can constantly change, requiring an adaptive network for best energy efficiency. For this reason, a paradigm shift is necessary to address all of these challenges and limitations by creating an efficient routing protocol.

In this paper, we propose Tracer assisted Passive Named Data Routing (TPNDR), which uses tracers to form adaptive and robust forwarding tables based on data categorization. Our focus for this work is on introducing Named Data Networking (NDN) $[5,6,7]$ techniques to UWSN constraints. NDN uses certain concepts like naming data and routing towards these data types using a name query. This type of routing takes advantage of not needing an end address in order to route unlike IP-based routing. Instead each node can relay names in query towards unknown data locations. For TPNDR, we first use a preset name categorizing scheme for the data which is sensed and passively store on the local sensor node. Users can request data through the creation of interest packets at the surface buoy. These packets will then be transmitted throughout the network; with their targets being the queried data in request. Node routing tables are updated through a tracer scheme which is generated from the data sensed. This in turn creates a passive virtual path that can adapt over time based on changes of sensed data location and movement of relay nodes. To be more precise the goal of this routing scheme is for passive sensing in a large network, for example, oil or $\mathrm{CO}^{2}$ seafloor monitoring. By adapting NDN techniques we are able to achieve TPNDR's energy efficiency, while introducing certain features like multicasting and redundant packet removal. 
In order to fully analyze TPNDR we must compare some of its features to other protocols in the aspect of data source recognition and retrieval techniques. This arises two currently used schemes in UWSN; namely data mules and adapted flooding. Through extensive discussion and simulation we are able to show that we can locate the data in query and return it with fewer transmissions. This correlates to less energy used per node and, in general, a longer lifespan for the entire underwater network. Additionally, we ensure that even though information is stored passively, in the case of time critical data, we are still able to transmit to the surface. This type of underwater routing adapts a new perspective in using a relatively new concept of NDN in order to overcome the many complications of UWSN previously mentioned.

The remainder of this paper is structured as follows: Section II will discuss similar work to TPNDR and give an indepth look at some of their limitations. Next, we will give a general overview of NDN and highlight some of the key characteristics in Section III. Following this, in Section IV, will be TPNDR protocol design and implementation. Section $\mathrm{V}$ will consist of the simulation results. In Section VI, we present future work and conclude our work.

\section{RELATED WORK}

UWSN have received a large amount of research dedicated to their different forms of routing. Many of these protocols are adapted by the type of application and the water condition of the network. Some of the more common routing techniques are VBF [8], HH-VBF [9], DBR [10], MPT [11], and HydroCast [12]. These protocols use a broad range of data dissemination means like flooding, multipath and cluster based to transmit packets from the sensor to the surface node. The general idea for these approaches is pushing the data from the sensing source to the surface or receiver in an active approach in the shortest path form. This includes assumptions like the majority of the data being transferred on a first come order. Since our problem approach assumes that the location of the sensed data source(s) is unknown and varies over time, we choose flooding to be the most closely related routing protocol. In this case, we assume the network must flood the entire area or region of the UWSN in order to locate the single or multiple sensed data sources of query. Flooding approach incorporates a few crippling features for UWSN. The key idea behind flooding is trying all possible paths, which results in duplicate packets being transmitted across the network. For a large network, overtime, this can have a very dire effect in node battery replacement and packet collisions.

A more passive form of data routing is AUVs transporting data from underwater storage nodes to the target surface. In this way sensed data is stored locally on either the sensing node or a local cluster head. Periodically, an AUV will pass by either retrieving all or pieces of recently sensed data depending on querying descriptions. Since the AUV can move freely among the network, it has the capability to exchange data at very close ranges to help mitigate transmission delays. Once all necessary nodes are visited, the vehicle will return to the surface for data extraction. This, of course, creates unnecessary delays in data retrieval as well as additional complexity of what to fetch and how.

Some UWSN protocols are adapted from widely known, tested, and used ground-based networking techniques. One existing approach that relates to our work is direct diffusion [13]. In this area, we see certain techniques like path reinforcement or redirection based on adaptive and robust network analysis. Ground-based networks apply this technique by observing data paths on each node to anticipate future data paths. Reinforcement can allow more data to flow on a certain path based on energy awareness, congestion, and shortest distance. Direct diffusion disperses transmitted data if necessary by stressed nodes among wireless sensor networks. The three main shortcomings of this type of work in UWSN are the dependency on GPS localization, full flooding for path recognition, and asymmetric channel environments. While GPS can be substituted for other underwater localization schemes, flooding is still a major disadvantage. Reinforcement techniques weaken due to the harsh channel environments seen in underwater networks. Factors like marine mammals and large propagation delays in UWSN can lead to degraded acoustic spectrum usages, as seen in Luo et al. For these reasons, we look to NDN for inspiration in developing an energy efficient, adaptive routing protocol for UWSN.

\section{NAMED DATA NETWORK}

Van Jacobson et al. originally proposed NDN as a way to redesign the architecture of today's internet. NDN is a relatively new and upcoming field revamping the way we look at networking. In IP networking we have grown accustom to a source/destination focus. In contrast, NDN is data driven. NDN dynamically routes queries until satisfied by their target data, while duplicate data queries can be multicast. This is achieved through changing the end-to-end principle of the internet to introduce data driven connectivity.

NDN provides the idea of two routing tables, namely Pending Interest Table (PIT) and Forwarding Information Base (FIB). The PIT is designed around containing all current unsatisfied name queries and their corresponding neighboring node. Each entry in this table is accompanied with a timer to determine if the target data needs to be requested again. The FIB is designed to determine where the receiving packet should be forwarded in route to its requested data. In this way new entries are created for each unique name query. To remove duplicate name queries, the receiving packet's requester can be added to the existing entry. Certain fields like a stale timer, requester ID, round trip time, and the rate limit are also included in this entry. NDN also provides a form of caching on each node. This is called the Content Storage (CS) which contains previously fetched data for quicker routing hits in the future.

NDN incorporates two different types of packets: interest and data packets. Interest packets are generated by the consumer and contain a name to identify the data in query. By using the FIB, each node can distinguish where the interest packet needs to be sent in order to reach its intended data. It is important to note that in the case of ground-based networking, 
this does not imply the data producer but rather the closest known node with matching stored data. Once this interest packet is satisfied, a new packet, in this case a data packet, will be created with the corresponding information in query. An additional form of security is placed on the data packet in order to securely label the original producer of the content. Following the creation of this packet, nodes will redirect the packet back to the consumer. Nodes incorporate PIT to remove duplicates when receiving multiple interest packets of the same name. In this way, a node can create a list of all corresponding neighbors for a specific name and multicast the data packet once the query has been satisfied. To facilitate the movement and caching of data, each node stores recently received data in its local CS.

The design of this protocol assumes a form of hierarchically structured names as a replacement to IP network's IP addresses. One of the main attributes for this is enabling relationships between pieces of data (e.g. /uwsn/images/high_res/marine_life/2/4). Another interesting feature is the use of congestion scaling on each node. Similar to direct diffusion's reinforcement, NDN nodes are able to limit or stop sending interest packets to a specific neighbor depending on incoming data traffic. With the vast differences between ground-based wired networks and UWSN, not all of these features remain relevant when constructing our protocol around NDN. We still hold the overall idea of NDN and its promising characteristics to demonstrate energy efficiency among an underwater network.

\section{TPNDR PROTOCOL DESIGN}

In this section we break down the different aspects of our UWSN protocol. In part, we adapt certain features of NDN in order to fully optimize routing among a passive data storing network.

\section{A. Overview}

The focus of this work is monitoring a set of different variables which exceed the transmission limitations of the network. An example of this could be a seafloor $\mathrm{CO}^{2}$ monitoring system which is comprised of multi-variable sensing equipment. Additionally, this example system would have many sensing nodes spread among a large surface area, which collect large amounts of data over time. These sensor nodes would be spanned out among the seafloor in a large grid layout. Multiple relay nodes connect these sensors to the surface buoy through acoustic communication channels. From here the buoy has the ability to transmit in either RF, above ground, or acoustic for underwater. Since the sensor nodes will passively sense and store data on their local memory, the surface node must create a request in order for data to be transmitted up. Additionally, since so many nodes exist among the UWSN, we can assume that an optimal path must be acquired in order to reduce the energy usage for each data packet retrieved. To achieve this goal, we introduce multiple adapted concepts to TPNDR. These concepts are Pending Interest Table, Forward Information Base, Content Storage, naming conventions, and tracers.
In comparison to regular NDN protocol, we make a few changes to better match the underwater environment. Since the scope of our paper is on routing of information, we remove any form of security designed for ground based NDN. Next, we ignore possible collisions and other transmission issues since we assume other network layers will handle them. While UWSN are highly prone to transmission errors, our focus for this work is on the routing and retrieval of interest and data packets. To reduce packet size we introduce a bounded name entry in both interest and data packets. For interest packets, this bounding translates to limiting the name size to slightly reduce transmission costs. Data packets are bounded by size, in which large amounts of retrieved data will be split into chunks and transmitted to the surface buoy in sequence.

\section{B. Adapted Pending Interest Table}

In order to fully integrate the characteristics of NDN, we must first define the functionality and fields of the PIT in an UWSN for TPNDR. Similar to ground-based NDN, the PIT will keep track of all name entry requesters and next hops. Fig. 1 depicts these entry fields. A PIT entry will contain a list of all known interest names. Linked to these entries will be a corresponding requester ID and timestamp, followed by all outgoing next hops. For all interest names there exists a next hop neighbor ID. This next hop can either directly reach the originating data source or move the interest packet towards the data source. Since the underwater environment entails many additional hazards among acoustic channels (e.g. marine-life, acoustic reflection or refraction, mobility, etc.), we must also address packet loss. A send-timer is incorporated with the outgoing next hops to help remove channel instability through a rebroadcast feature. This feature is activated once this sendtimer expires for the individual entry.

Fig. 2 depicts the general flow of a node receiving an interest packet. Only sensor nodes store data locally. With this assumption, only sensor nodes will check their content

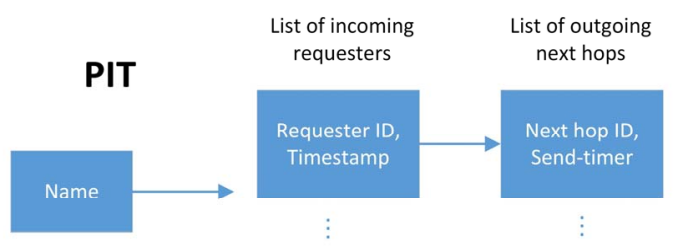

Fig. 1. Pending Interest Table (PIT) entry fields.

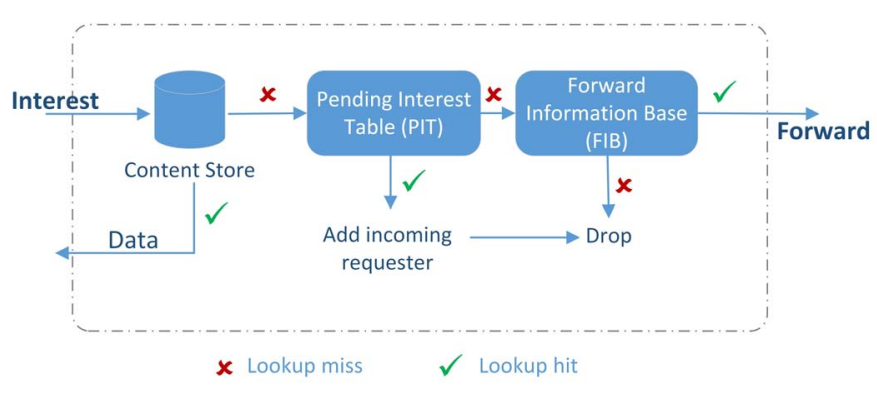

Fig. 2. Node handling scheme for interest packets. 
storage for matching data. All other nodes will bypass this step (as described in subsection D of Section IV). If the node's PIT lookup for the interest is found, we add the requester to the related name entry and drop the packet. Therefore a previous interest has already been sent out among this path and we can remove this duplicate packet. If no entry exists we must check that the FIB has a previously known entry for this specific name query. If not, we know that the node does not reside along this path and therefore can drop the packet. Only if the FIB forwards to the next hop do we update the PIT.

\section{Adapted Forwarding Information Base}

The FIB consists of a relatively simple forwarding table, similar to ground based NDN. Fig. 3 depicts a general idea of FIB's table and how it handles its entries with incoming data packets. FIB entries consist of names and their related next hop neighbors. An entry's next hop neighbor is the next node, within broadcast distance of the sender, which is one hop closer to the data source of interest. It is important to note that these entries are updated through the use of tracers (which will be described in subsection F of Section IV). Each next hop neighbor consists of a stale time to ensure that the data along the path has not gone stale. This feature, in collection with tracers, allows a robust and adaptive approach to data location and path development.

Fig. 4 illustrates the process a node takes when receiving a data packet. In this case we first check to see if there is a previous unsatisfied entry in PIT. If there are no entries related to the retrieved data packet, the node does not belong among the retrieval path of the packet. If an entry does exist, we update the FIB's stale time, round trip time (RTT), and status corresponding to the packet's table entry, before removing the outgoing next hop ID from the PIT. In this instance RTT represents the time it takes from interest forwarding to data packet retrieval. The RTT value helps better adjust the stale time based on congestion of the network. The status field indicates any possible dead or low energy nodes in the selected path. This value could prove to be useful in the case of a path with possible faulty nodes, and therefore will be looked into in future works.

Since each entry can potentially have multiple next hop neighbors, we see that FIB provides the ability for multiple sections of a network to process similarly categorized data. These multiple sectors can all be accessed through a single interest packet. We plan on fully addressing oversaturation of a single unique sensed data name in future work.

In the case where a node should die before satisfying its PIT entry, the above node will resend its interest packet to discover a new path to the data source once its entry has timed out. Next hop ID is used among packet transmission to ensure looping does not occur between two neighboring nodes. Additionally, larger routing loops cannot occur among interest or data packets due to the removal of a packet when a duplicate or missing PIT entries occurs.

\section{Content Storage}

Caching on each node's local content storage in groundbased NDN is a critical process in improving current internet structures. However, for UWSN, we see this type of data processing as unnecessary and inefficient. For our environment this caching complexity becomes a two-fold issue for network nodes: namely storing stale data and wasting node storage space. Since we know that nodes must use acoustic communication among UWSN, it only makes sense to remotely store the previously fetched data on a device with radio frequency capabilities. This means we can theoretically store the fetched data either on the surface buoy or at a remote ground-based database. For constraint purposes, we assume that once the data in query has reached the surface it has surpassed the underwater challenges and can furthermore be routed with ease. Since UWSN nodes are resource constrained it would be meaningless to store this data after the next hop node (towards the surface) has already received the requested data. In this way we can assume that network caching, on relay nodes, will not be used. It is important to note that sensor nodes do have a content storage in order to locally categorize and store data. The means of storing data, once memory has been exhausted, is an application based issue. For our general case, we can assume a First-In First-Out caching policy.

\section{E. Naming Convention}

Due to the definition of an interest packet, we organize data into naming conventions in order to be routed properly. To achieve this we must break sensed data into naming types. This naming convention is preset by the application but can be reset with a general message to all nodes, if need be. In order to best illustrate this we will give an example with three different viewpoints as follows:

(1) Extrem_High_Temp

(2) $/$ Sensor $2 /$ Temp/04_14_2014/Extrem_High

(3) /Node1/Node2/Sensor $2 /$ Temp/04_14_2014/Extrem_High
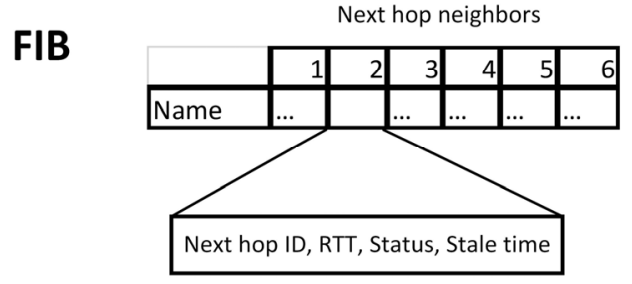

Fig. 3. Forward Information Base (FIB) entry fields.

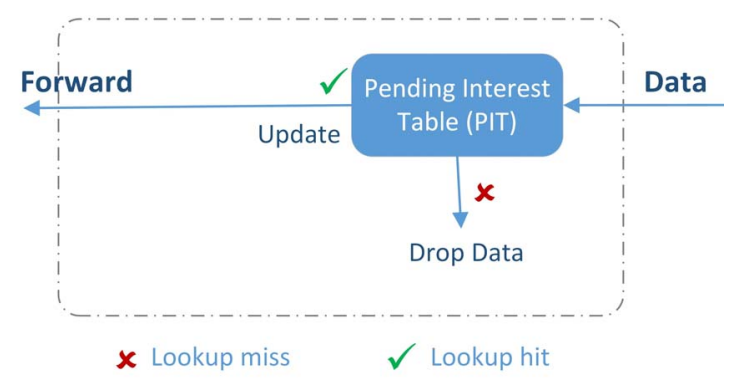

Fig. 4. Node handling scheme for data packets. 
In this example (1) is the generic name of the data, which could group all data sensed above $50^{\circ} \mathrm{F}$. Next would be the sensor's internal view (2) and finally the entire path (3). While the entire path name is never used, it is given as an example to see the path traverse along the network. Each sensor node categorizes its collected data on an internal grouping system based around the preset naming convention. In this way, a given node can try to match its received interest packet's name with its internal view. Additionally, this internal view allows nodes to categorize either their $\mathrm{CS}$ data or sensed data downstream in a more precise manner. For example, relay Node2 would have an internal entry as /Node2/Sensor2/Temp/Extrem_High. On receiving an interest packet related to extremely high temperature, Node 2 would direct the packet towards Sensor2.

By using this naming convention, interest packets can become more flexible. Instead of specifying a certain name, these packets can instead search for a group of data. A data size limit can be placed on this request in order to not overload the network with one grouping of data. Additionally, since data packets are bounded, we are able to break the information into chucks on retrieval while still routing in the same fashion.

\section{F. Tracers}

In the case of data location being unknown to the network, current work has substituted different types of flooding to compensate. As previously mentioned, this type of technique wastes a great amount of energy especially in a large network. For this case, we propose using passive tracers to form virtual paths from the data sense location to the surface. These tracer packets will be small in size, consisting of the target name being passed, the ID of the sender, and the original sensed data timestamp. The general idea of this is for the tracers to have little impact on transmissions and energy costs. Tracers are defined as single directional communications toward the surface buoy. Certain features, such as when tracers are sent out and how they are handled by nodes must be addressed.

For each naming convention group on a node, a threshold will be in place to determine when a tracer should be broadcasted. In this manner, we can withhold a flood of tracers while still ensuring that this virtual path from the data source to the surface is created. Each threshold is name category specific to allow different paths to form from different values being sensed. The origin of the tracers is from the sensor nodes, which increments a counter towards the threshold for each newly sensed data. For each occurrence of a node receiving a tracer packet, the stale timer of the related name entry on the node's FIB will be reset. In this way, the tracer defines the path as still fresh.

Any UWSN node, excluding the surface buoy(s), will recursively repeat this process. Over time this will create virtual paths from the sensing source all the way to the surface with correlation to the type of data being sensed. This allows higher frequency data to be adapted into the network, while ignoring outliers that could appear. Additionally, this can help remove certain issues that could arise from random incorrect data sensing. It is important to note that a priority system could be implemented in the case of highly critical information being sensed and automatically pushed towards the surface.

On receiving a tracer or data packet, a node will update its FIB in relationship to the data name and the node neighbor it was sent from. In this way, we can find trending data paths as well as ensure past paths still contain fresh data. In the case that nodes should fail or if data is not sensed in a certain location of the network, once stale timers have been reached, the corresponding path will be removed. Therefore tracers create an adaptive and robust approach to passively locating UWSN data.

Based on the preset threshold on each node and the frequency of sensing, tracers will take time to fully populate the network. To overcome this issue we introduce a learning phase at the beginning of the routing protocol. By reducing all nodes' threshold for this short timeframe, we can create multiple paths. This will ensure that the UWSN will start mapping trending paths, pending application needs, which can change and adapt overtime. We like to note that if instantaneous routing paths are unnecessary this learning phase can be ignored by setting pre and post phase thresholds to the same value.

\section{Simulation RESUlts}

We conducted experimental tests to evaluate the energy performance and specific thresholds that are necessary in improving and validating TPNDR. Due to NDN being a relatively new field we had difficulties finding other work to compare our proposed protocol with. For this reason, we choose to compare TPNDR, in an identical network layout, to an adapted flooding protocol. While our data fetching approach is not typical for regular routing techniques, adaptive flooding still holds relevance in our scenario.

\section{A. Simulation Settings}

We constructed our simulation to mimic underwater transmission delays and packet dissemination. The simulator was written in $\mathrm{C}++$ programming language as a standalone program. The layout of our simulated network consists of 22 nodes in a $10 \mathrm{~km} \times 4 \mathrm{~km}$ area, with one surface buoy, 11 relay nodes, and 10 sensor nodes. For simulation purposes these sensor nodes represent a cluster of many nodes spread out in a mesh grid on the seafloor. Since we are looking at clusters of sensors we demonstrate our simulation in a two dimensional scope. Additionally, we assume gaps between nodes of no more than $1 \mathrm{~km}$ in size, to keep transmission range in reasonable distances. No restriction was placed on the sizes of tables or memory buffer, since we can assume the following: names are of bounded preset size, only next hop neighbor is viewable, and sensing type is limited. Name convention was constrained to different $\mathrm{CO}^{2}$ and temperature readings.

\section{B. Effects of Threshold Value}

In order to verify TPNDR, we first have to look at the threshold value and how it corresponds to the variation of transmissions in the entire network. By reducing the amount of transmissions, we can assume that overall energy usage is directly affected. In order to depict the learning phase of the 
network, we first start all nodes with a threshold of one. This learning phase lasts for 1,000 seconds. We choose this value since it roughly estimates how long it takes to construct a full tracer path from a sensor node to the surface. This value can vary depending on the size, depth, sensing frequency and density of the network, but nonetheless does not need to fully populate a path to the surface. Once the learning phase is complete, all nodes reset their threshold to their preset value.

In Fig. 5 we show our results of changing this threshold value and its effect on transmissions over a 10,000 second simulation run. Sensing occurs every 200 seconds on each sensor node, with the most frequent value being sensed as name Med_CO ${ }^{2}$. As apparent, there is a steep drop off at value 2 for threshold. This illustrates the implementation of the threshold value changing once the learning phase has completed. It is interesting to note that without this threshold value being more than 1 , it behaves similarly to a regular UWSN protocol in which all data collected is sent to the surface. After threshold value 5, there is relatively little change in total transmissions. While this example simulates a span of a little less than three hours, it is assumed that the goal of an UWSN is to endure months to years without much intervention. As previously mentioned this threshold value is not necessary but instead institutes the ability to populate node's PIT in a passive network.

\section{Effects of Name Categorization}

To determine the effect of poor name categorization, we next look at the amount of unique name values in relationship to total transmissions for TPNDR. In this scenario we assume all sensor nodes are of equal distance to the surface buoy. In the worst case circumstance every sensor node has a path to the surface, created from passive tracers of unique data types. For this work we removed the learning phase and ran the simulator for 30,000 seconds. Each node's sensing frequency was set at 60 seconds. We ran this study to see if the reduction of duplicate tracers would adapt differently with changes in data type sensed. Additionally we use this work to show how the use or misuse of the naming convention can be critical in energy efficiency. As seen in Fig. 6, there is a relatively linear change based on how many unique name categories are being

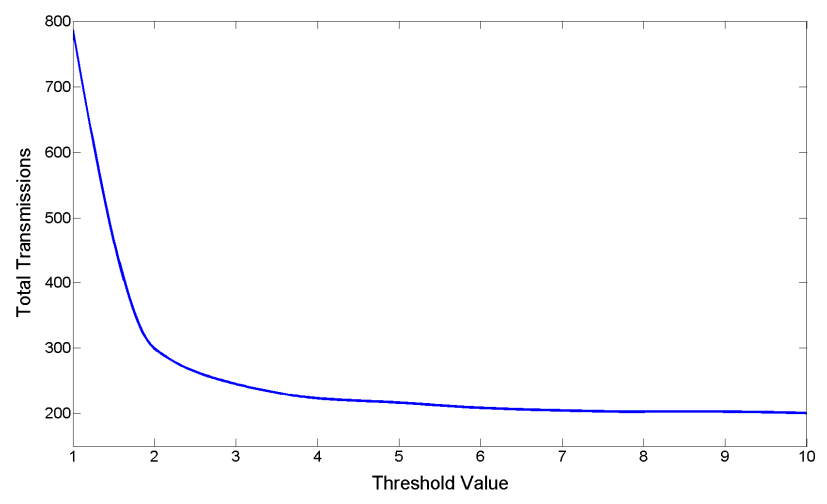

Fig. 5. Energy consumption vs. threshold value for TPNDR protocol. This threshold value is implemented after the learning phase halts. sensed at once. This is most likely due to the tracer threshold on each node being set to value 5 , which highly reduced the effectiveness of removing duplicate tracers. On this scale only small differences can be seen from traffic volume, however we believe that there will be larger changes in data values as experimental times grow. For this reason, we leave this topic for future works.

\section{Effects of Network Size}

We next analyze how the size of the network will affect each protocol in terms of energy usage. In order to accomplish this we run each protocol for 20,000 seconds at varying network sizes and record the amount of transmissions. We assume consistent interest requests and sensing periods. Since we are looking at the work in a two dimensional way, we assume that sensor node clusters are distributed evenly throughout the previously described network. In Fig. 7 we compare TPNDR with adaptive flooding and demonstrate our findings. It is clear that after we add more than four sensor node clusters, or in other words two clusters to each side of the seafloor scenario, TPNDR outperforms flooding in total transmissions. This demonstrates that an adaptive flooding protocol may be efficient in a small passive network, but once you expand the sensed region, adaptive flooding quickly becomes a waste of node resources. It is important to note that the amount of relay nodes and the buoy stay static in this scenario.

\section{E. Energy Consumption}

For the comparison of the adaptive flooding protocol we assumed the same simulated network was in place. The goal is searching for sensed data, with an unknown location, and returning one or multiple collection(s) of said data. In order to make this flooding protocol more relevant we constrained it to only flood certain sectors of the network and slightly adapt to changes in data sensed. In this flooding technique nodes are given the ability to recognize where previously located data was sensed. Flooding can occur in sectors of the network thus eliminating constant flooding throughout the UWSN. One large assumption we made is not allowing previously located data to drastically change location. This means that data types

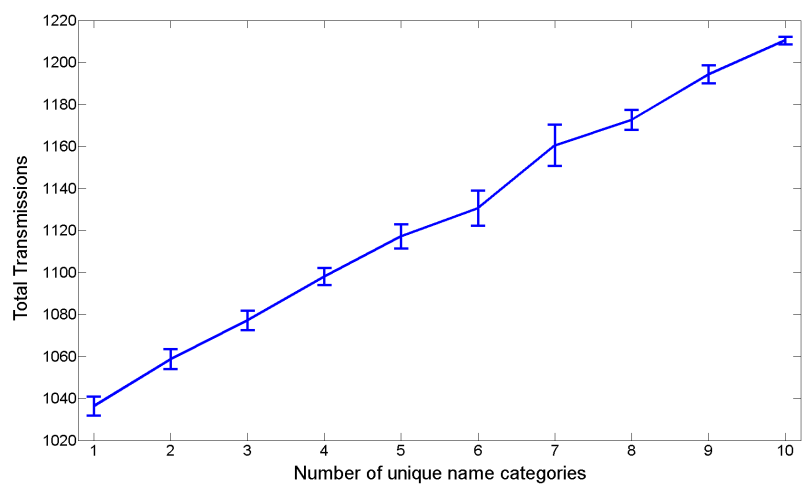

Fig. 6. Energy consumption based on number of unique name categories preset for TPNDR protocol. 
were constrained by network sectors for this adaptive flooding protocol.

In order to compare this adaptive flooding protocol to TPNDR we looked at the total transmissions over a period of time. This illustrates how much energy each protocol uses based on periodically requesting data from the surface buoy. We also want to note that due to the small size of tracers, we examined the overall cost of transmissions if we assume tracers are half the size of regular packets. We can see in Fig. 8 that TPNDR outperforms an adaptive flooding protocol. Since we assume that our tracer packets are much smaller than other interest and data packets we also depicted another line (TPNDR v2) to show the difference.

\section{F. Performance}

In order to determine the performance of TPNDR we turn our focus to the time it takes for sensed data to create a tracer path to the surface buoy. In this way we can view the adaptability of TPNDR in comparison to changing of sensed data type over time. To determine this comparison we mark every time a tracer reaches the surface buoy, which depicts that the virtual path still holds true. This process, at the surface, will either form a new path or refresh an already standing one before the stale timer is met. For our experiment we set stale timers as slightly above the trip time of a packet from the source node to the current node. We also introduce outlier sensed data, which only occurs during a limited timeframe, and for this reason, never forms a passive path to the surface. For this scope, we have six different forms of data categorization. For simplification, we assume each sensor cluster can have at most one unique name being sensed at a time. This translates to, at most, ten possible paths which can be connected to the surface buoy at once. In Fig. 9 we fully depict this experiment. In this figure, markers show when data is first available to be sensed, and repeated markers show when this sensed data availability ends. Sensing occurs for all node clusters in frequency of $0.1 \mathrm{~ms}$. All nodes go through an original learning phase which lasts for $1000 \mathrm{~ms}$ in the beginning of simulation, followed by returning to a tracer threshold of 3. Stale timers vary based on location of relay nodes; for instance, the surface buoy's stale timers are set to $10 s$. For comparison, we include a flooding protocol which based on the nature of flooding forms a path to every sensor node. In the figure we notice a slight delay, for TPNDR, in when sensed data is available and when the full path to the surface is formed. This is based around passive tracers forming and tracer thresholds on each next hop. It is also important to see that Sensed Data 3 never connects to the surface, due to being available for too short of a timeframe. This path formation and removal shows TPNDR's adaptive nature in connection to sensed data type over time.

\section{CONCLUSION AND FUTURE WORK}

In this paper, we identified a UWSN routing protocol, named Tracer assisted Passive Named Data Routing (TPNDR), which uses NDN conventions and passive tracers to fetch data in an energy efficient manner. By analyzing ground based wired NDN, we adapt certain key principles to

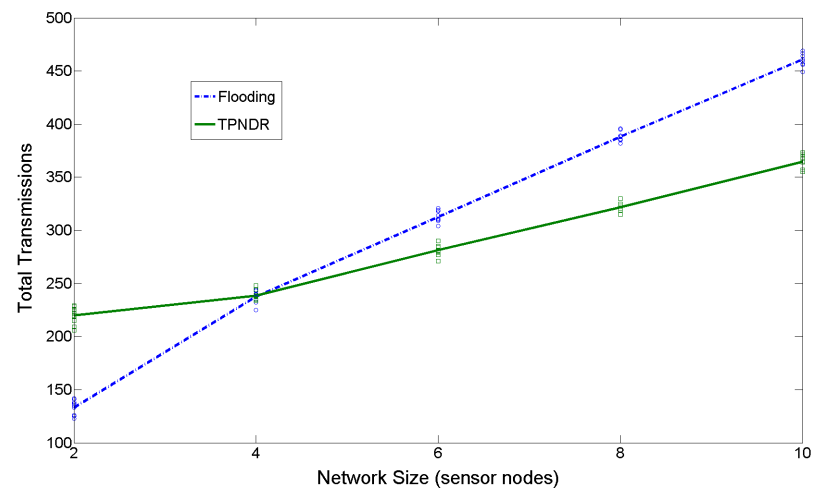

Fig. 7. Energy consumption vs. network size in amount of sensor node clusters.

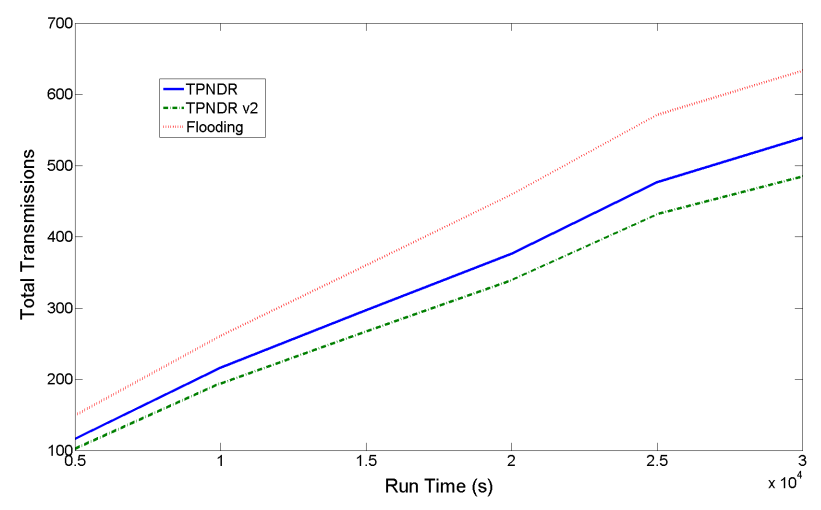

Fig. 8. TPNDR protocol compared with adaptive flooding protocol in terms of total transmissions sent.

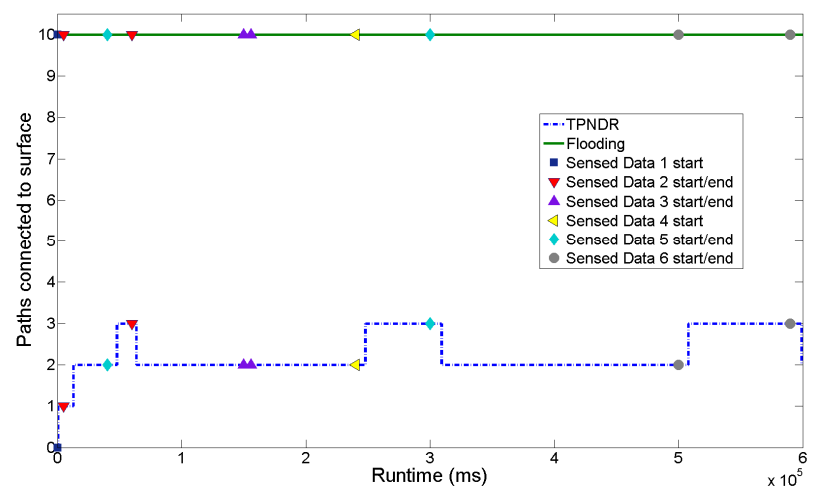

Fig. 9. Amount of paths connected from sensed data at seafloor to surface buoy. Markers illustrate start and end of data availability.

improve the many challenges faced in underwater. Additionally, we look at the idea of a passive sensing environment, in which large amounts of data are collected over time and only specifically requested information is returned to the surface buoy. In this fashion we integrate passive tracers to adaptively create paths from the sensed 
source to the surface through stale data timers and data frequency thresholds. These routing techniques prove to reduce redundant packets as well as add robustness against the harsh underwater environments. Through experimentation we are able to verify the effectiveness of tracers among our routing protocol. We also compare our work against an adaptive flooding protocol in focus of transmissions needed to discover and retrieve specific data. Our name based protocol proves to be more efficient in this scope, thus portraying a more energy efficient routing scheme.

While our evaluation purposes focused on the standard protocol scheme in a condensed view, TPNDR could easily be expanded to a much larger network. In this way the basic improvements seen could notably increase, and therefore greatly extend the lifespan of the network and its data transfer reliability. By creating a more node dense and larger network, it would be interesting to see the varying phenomenon that may appear. In this work, we made the assumption of a fully connected network, while it would be interesting to analyze the effect of possible routing holes. This idea would develop new routing issues which must be considered.

In order to address the case of overly common name classifications overwhelming the network, we could incorporate varying tracer thresholds depending on name type. This solution implies many assumptions and therefore requires future research to demonstrate its strength and weaknesses. Since simulations can only depict a limited viewpoint, we also plan on conducting lake and sea field tests in order to evaluate and further develop TPNDR.

\section{REFERENCES}

[1] Partan, J.; Kurose, J. \& Levine, B. N. (2006), A Survey of Practical Issues in Underwater Networks, in 'Proc. WUWNet', pp. 11-24.
[2] Akyildiz, I. F.; Pompili, D. \& Melodia, T. (2005), 'Underwater Acoustic Sensor Networks: Research Challenges', Ad Hoc Networks 3(3), 257281.

[3] Cui, J.-H., Kong, J., Gerla, M. and Zhou, S. "Challenges: Building Scalable Mobile Underwater Wireless Sensor Networks for Aquatic Applications," IEEE Network (20:3), 2006, pp. 12--18.

[4] Luo, Y., Pu, L., Zuba, M., Peng, Z. and Cui, J.-H. "Challenges and Opportunities of Underwater Cognitive Acoustic Networks"(UbiNetTR13-10), Technical report, UCONN CSE, 2013.

[5] Jacobson, V., Smetters, D. K., Thornton, J. D., Plass, M. F., Briggs, N. H. and Braynard, R. L. "Networking Named Content"'Proceedings of the 5th International Conference on Emerging Networking Experiments and Technologies', ACM, New York, NY, USA, 2009, pp. 1--12.

[6] Zhang, L., Estrin, D., Burke, J., Jacobson, V., Thornton, J., Smetters, D. K., Zhang, B., Tsudik, G., kc claffy, Krioukov, D., Massey, D., Papadopoulos, C., Abdelzaher, T., Wang, L., Crowley, P. and Yeh., E. "Named Data Networking (NDN) Project"(NDN-0001), 2010.

[7] Yi, C., Afanasyev, A., Moiseenko, I., Wang, L., Zhang, B. and Zhang, L. "A Case for Stateful Forwarding Plane," Comput. Commun. (36:7), 2013, pp. 779--791.

[8] Xie, P., Cui, J.-H. and Lao, L. "VBF: Vector-Based Forwarding Protocol for Underwater Sensor Networks"(Technical Report: UbiNet-TR05-03 (BECAT/CSE-TR-05-6)), Technical report, UCONN CSE, 2005.

[9] Nicolaou, N., SEE, A., Xie, P., Cui, J.-H. and Maggiorini, D. "Improving the Robustness of Location-Based Routing for Underwater Sensor Networks"'OCEANS 2007 - Europe', 2007, pp. 1-6.

[10] Yan, H., Shi, Z. and Cui, J.-H. "DBR: Depth-Based Routing for Underwater Sensor Networks"'Proc. IFIP Networking', 2008, pp. 1--13.

[11] Zhou, Z. and Cui, J.-H. "Energy Efficient Multi-path Communication for Time-critical Applications in Underwater Sensor Networks"'Proceedings of the 9th ACM International Symposium on Mobile Ad Hoc Networking and Computing', ACM, New York, NY, USA, 2008, pp. 221--230.

[12] Lee, U., Wang, P., Noh, Y., Vieira, F., Gerla, M. and Cui, J.-H. "Pressure Routing for Underwater Sensor Networks"'INFOCOM, 2010 Proceedings IEEE', 2010, pp. 1-9.

[13] Intanagonwiwat, C., Govindan, R. and Estrin, D. "Directed Diffusion: A Scalable and Robust Communication Paradigm for Sensor Networks"'Proceedings of the 6th Annual International Conference on Mobile Computing and Networking', ACM, New York, NY, USA, 2000, pp. 56--67. 